\title{
Maternal Hyperglycemia Directly and Rapidly Induces Cardiac Septal Overgrowth in Fetal Rats
}

\author{
Erin E. Gordon, ${ }^{1}$ Benjamin E. Reinking, ${ }^{1}$ Shanming Hu, ${ }^{1}$ Jianrong Yao, ${ }^{1}$ Kok L. Kua, \\ Areej K. Younes, ${ }^{1}$ Chunlin Wang, ${ }^{1}$ Jeffrey L. Segar, ${ }^{1}$ and Andrew W. Norris ${ }^{1,2,3}$ \\ ${ }^{1}$ Department of Pediatrics, University of Iowa Carver College of Medicine, Iowa City, IA 52242, USA \\ ${ }^{2}$ Department of Biochemistry, University of Iowa Carver College of Medicine, Iowa City, IA 52242, USA \\ ${ }^{3}$ Fraternal Order of Eagles Diabetes Research Center, University of Iowa, Iowa City, IA 52242, USA \\ Correspondence should be addressed to Andrew W. Norris; andrew-norris@uiowa.edu
}

Received 9 December 2014; Revised 28 March 2015; Accepted 22 April 2015

Academic Editor: Christoph H. Saely

Copyright (C) 2015 Erin E. Gordon et al. This is an open access article distributed under the Creative Commons Attribution License, which permits unrestricted use, distribution, and reproduction in any medium, provided the original work is properly cited.

\begin{abstract}
Cardiac septal overgrowth complicates $10-40 \%$ of births from diabetic mothers, but perplexingly hyperglycemia markers during pregnancy are not reliably predictive. We thus tested whether fetal exposure to hyperglycemia is sufficient to induce fetal cardiac septal overgrowth even in the absence of systemic maternal diabetes. To isolate the effects of hyperglycemia, we infused glucose into the blood supply of the left but not right uterine horn in nondiabetic pregnant rats starting on gestational day 19. After $24 \mathrm{~h}$ infusion, right-sided fetuses and dams remained euglycemic while left-sided fetuses were moderately hyperglycemic. Echocardiograms in utero demonstrated a thickened cardiac septum among left-sided (glucose-exposed, $0.592 \pm 0.016 \mathrm{~mm}$ ) compared to right-sided (control, $0.482 \pm 0.016 \mathrm{~mm}$ ) fetuses. Myocardial proliferation was increased $1.5 \pm 0.2$-fold among left-sided compared to rightsided fetuses. Transcriptional markers of glucose-derived anabolism were not different between sides. However, left-sided fetuses exhibited higher serum insulin and greater JNK phosphorylation compared to controls. These results show that hyperglycemic exposure is sufficient to rapidly induce septal overgrowth even in the absence of the myriad other factors of maternal diabetes. This suggests that even transient spikes in glucose may incite cardiac overgrowth, perhaps explaining the poor clinical correlation of septal hypertrophy with chronic hyperglycemia.
\end{abstract}

\section{Introduction}

Cardiac septal overgrowth affects $10-40 \%$ of neonates born to pregnancies complicated by maternal diabetes [1-6]. The functional impact of neonatal cardiac septal hypertrophy can range from clinically asymptomatic to potentially fatal congestive heart failure stemming from left ventricular outflow tract obstruction. Neonatal cardiac septal hypertrophy (meaning overgrowth) is rare among infants from nondiabetic pregnancy $[5,7]$, and diabetes during pregnancy imparts an 18-fold relative risk compared to nondiabetic pregnancy [6].

It is unclear what component of the intrauterine milieu perturbed by maternal diabetes is responsible for fetal cardiac septal overgrowth. Because of the close link to maternal diabetes, hyperglycemia is a leading causative candidate. However, the relationship of maternal glycemia to neonatal cardiac septal hypertrophy remains uncertain. Some studies report that elevated maternal glycemia is associated with cardiac septal hypertrophy $[3,8]$, whereas other studies report no relation $[2,4,5,9,10]$. Furthermore, newborns from pregnancies where glucose levels have been tightly controlled are still at risk of septal hypertrophy [11-13]. More surprisingly, newborns of tightly controlled diabetic mothers still have a high incidence of cardiac septal hypertrophy despite having abnormally low glycosylated fetal hemoglobin $\left(\mathrm{HbF}_{1 \mathrm{c}}\right)$ indicating lower than normal antecedent fetal glucose levels [14]. Because of the uncertain relation between hyperglycemia and neonatal cardiac septal hypertrophy, it has been postulated that other derangements of diabetic pregnancy, such as other elevated maternal fuels, altered placental nutrient transport, or hormonal disturbances, may be responsible for the septal overgrowth $[2,6,15]$. 
To better understand the interaction between glucose and the fetal myocardium, our laboratory has utilized a recently established rat model $[16,17]$ that delivers localized hyperglycemia to selected fetus in nondiabetic dams. Using this model of maternal diabetes, we examined the effects of hyperglycemia on the fetal myocardium during late gestation. Importantly, this model isolates the effects of hyperglycemia only, avoiding the myriad effects of maternal diabetes since the dams and control fetuses remain euglycemic throughout $[16,17]$. We used this model to test the hypothesis that hyperglycemia is sufficient to induce fetal cardiac septal hypertrophy, finding that even transient hyperglycemic exposure induces septal overgrowth. Because this result directly implicates maternal hyperglycemia in the etiology of septal overgrowth, we examine signaling pathways and gene expression patterns that might contribute to glucose-related overgrowth, finding potential roles for fetal hyperinsulinemia and for JNK activation.

\section{Materials and Methods}

2.1. Animals. All animal procedures were performed within the regulations of the Animal Welfare Act and the National Institutes of Health Guide for the Care and Use of Laboratory Animals and were approved by the Institutional Animal Care and Use Committee of the University of Iowa. Timedpregnant Sprague-Dawley nondiabetic female rats (Hsd: Sprague Dawley SD; Harlan Laboratories, Inc., Indianapolis, IN) were maintained on standard laboratory chow and water ad libitum. Gestational day 0 was defined as the day of initial vaginal plug detection and the normal gestational length for this strain is $21 \pm 1$ days. On the 19th day of gestation, an infusion catheter was placed as described [16] so as to expose only the fetuses of the left, but not right, uterine horn via the left uterine artery. Glucose $(20 \mathrm{~g} / \mathrm{dL})$, containing $2 \mathrm{U} / \mathrm{mL}$ heparin, was then infused continuously at $4 \mathrm{mg} / \mathrm{min}$ via this catheter at a fluid infusion rate of $20 \mathrm{microliters} / \mathrm{min}$. After 24 hours of infusion (i.e., on gestational day 20), fetuses were accessed for echocardiography or blood sampling while continuing the glucose infusion during these procedures. Fetuses were accessed by uterine exteriorization under warm, moist conditions via a midline abdominal incision under surgical anesthesia. Fetuses were studied in a fashion alternating between the left and right uterine horns beginning with those closest to the cervix. Fetal blood sampling was performed via incision of the right subclavian artery with collection into heparinized glass capillary tubes. Immediately following study, anesthetized fetuses were delivered by cesarean section and euthanized. A portion of the fetal hearts was rapidly excised to be frozen in liquid nitrogen and stored at $-80^{\circ} \mathrm{C}$ or placed in $1 \%$ formalin.

2.2. Echocardiography. Echocardiograms were performed using the Vevo 2100 High Resolution Imaging System and software (VisualSonics Inc., Toronto, Canada). The transducer was placed directly on the uterine wall at the level of each fetus, and the fetal heart was visualized via short axis view of the right and left ventricles. Diastolic thickness of the interventricular septum was measured at the level of the left ventricular papillary muscles. Each ultrasound thickness reported is the mean of 3 consecutive measurements. The measurements were made in accordance with the American Society for Echocardiography Guidelines by a single operator who was blinded as to the nature of the infusate.

2.3. Assays. Blood glucose was determined using a OneTouch (LifeScan, Milpitas, CA) meter. Serum was stored at $-80^{\circ} \mathrm{C}$ and later assessed for insulin content by ELISA (\#101250-01, Mercodia, Uppsala, Sweden).

2.4. Growth-Related Kinase Signaling. Several intracellular kinases are proposed to be signaling mediators in the pathogenesis of cardiac overgrowth. Of these, AKT and the MAPkinases JNK, ERK, and p38 are leading candidates [1821]. These were assessed by Western blotting of fetal heart homogenized using a MicroGrinder (RPI, Mount Prospect, IL) in $100 \mu \mathrm{L}$ cell lysis reagent (Sigma: \#C2978, St Louis, MO), $1 \mu \mathrm{L}$ protease inhibitor cocktail (Sigma: \#P8340), and $1 \mu \mathrm{L}$ phosphatase inhibitor cocktail set II (Calbiochem/EMD Biosciences: \#524625, San Diego, CA). Antibody immunoassays were performed for total and phosphorylated JNK, total and phosphorylated AKT (Cell Signaling Technology, Danvers, MA), and total and phosphorylated ERK. Band densities were quantified by V750 pro Scanner (Epson, Long Beach, Ca) and IMAGEJ. For Western blotting, in addition to 24-hour infused dams, a separate cohort of dams was studied, which underwent glucose infusion using an identical approach except that the infusion duration was 48 instead of 24 hours, being initiated on gestational day 18 and completed on gestational day 20 .

2.5. Cardiac Immunofluorescence. Cardiac tissues were fixed in $10 \%$ neutral buffered formaldehyde for 24 hours. Dehydrated sections were cut at $8 \mu \mathrm{m}$ thickness, placed on positively charged microscope slides, dried, deparaffinized with xylene, and rehydrated with graded ethanol. For antigen retrieval, slides were washed twice with $\mathrm{PBS}$, boiled for 20 minutes in $0.1 \mathrm{M}$ Sodium citrate buffer ( $\mathrm{pH} \mathrm{6.0)}$, and then permeabilized with $0.2 \%$ Triton X-100. Bovine serum albumin, 2\%, in PBS was used as a blocking agent. Slides were incubated overnight with $100-120 \mu \mathrm{L}$ rabbit anti-Ki67 antibody $(1: 50)$ at $4^{\circ} \mathrm{C}$. After washing in PBS, Alexa Fluor 488-conjugated secondary antibody $(1: 200)$ was added and nuclei were stained with To-PRO-3 (1:2000). Slides were examined at $40 \mathrm{x}$ magnification with a Zeiss 510 confocal microscope, 3 fields per slide. Ratios comparing nuclei with active proliferation (Ki67 stained) to all nuclei (TO-PRO3 stained) per field were obtained via manual counting. These histopathologic quantifications were performed by one individual who was blinded as to the sample identities.

2.6. Measurement of Warburg Effect-Related Gene Expression. A prominent mechanism by which glucose can generate cellular growth is the Warburg effect [22-26]. The Warburg effect increases the flux of glucose carbons into anabolic pathways, thus supporting excess cellular growth. This effect is induced by changes in gene expression that favor specific 
TABLE 1: List of RT-PCR primers.

\begin{tabular}{lcllc}
\hline Gene & NCBI GeneID & Forward & Reverse & Amplicon size (bp) \\
\hline Glut 1 & 24778 & CTTTGGCAGGCGGAACTC & TTGCCCAGTTTGGAGAAACC & 84 \\
Actb & 81822 & TTCCTTCCTGGGTATGGAATCC & GGATGTCAACGTCACACTTCATG & 77 \\
$H k 2$ & 25059 & TGGGCTGGACAACCTCAAAG & CCTTGGCAAAGTGAGGATGAAG & 74 \\
$P d k 1$ & 116551 & GCGAGACGGCTTTGTGATTTG & CCTGGTGATTTCGCATTTAGTTC & 77 \\
$P 53$ & 24842 & CACTCCAGCTACCCGAAGAC & GCCAGGAACCAGTTTGCATAG & 259 \\
L dha & 24533 & AATGAAGGACTTGGCTGATGAG & GCCATGCTGAAGATCCATCATC & 85 \\
Tigar & 502894 & ACGCCTTCTCCAGTGATCTC & CGTACATCCTTTCCCGAAGTC & 120 \\
Gpt2 & 307759 & CCCACAGGCCAGGTACAAAG & AAAGCTTCTCTTCCCAGGCAAAG & 70 \\
$P k m 2$ & 25630 & TGACACCTTCCTGGAACACATG & GGGAAGCAGGGCCAATGG & 98 \\
\hline
\end{tabular}

Gene abbreviations: Glut1, solute carrier family 2 (facilitated glucose transporter) member 1; Actb, beta actin; Hk2, hexokinase 2; Pdk1, pyruvate dehydrogenase kinase isozyme 1; P53, tumor protein p53; Ldha, lactate dehydrogenase A; Tigar, fructose-2,6-bisphosphatase TIGAR; Gpt2, glutamic pyruvate transaminase (alanine aminotransferase) 2; $P \mathrm{~km} 2$, pyruvate kinase muscle.

aspects of anaerobic glucose metabolism. Maternal diabetes favors Warburg conditions by both increasing glucose levels and further reducing fetal oxygen tension $[27,28]$. We thus tested the expression of 8 genes known to be induced in the Warburg effect (Table 1). To accomplish this, fetal rat hearts were snap-frozen in liquid nitrogen and homogenized in $1 \mathrm{~mL}$ TRIzol (Invitrogen, Carlsbad, CA) for 2 minutes at $50 \mathrm{~Hz}$ on a LT TissueLyser using one $7 \mathrm{~mm}$ SS bead (Qiagen, Valencia, CA). RNA was isolated via chloroform extraction and isopropanol precipitation according to the TRIzol protocol. RNA, $1 \mu \mathrm{g}$, was served to synthesize cDNA using a high-capacity, random-primed reverse transcription kit (product \#4368814, Applied Biosystems, Foster City, CA) according to the manufacturer's instructions. RT-PCR primers were designed such that at least one of each primer pair spanned exon-exon boundaries using Primer3plus [29], avoiding amplicon's secondary structures using mFold [30] and avoiding dimer-primer and hairpin structures using Beacon Designer Free Edition (Premier Biosoft, Palo Alto, CA). Primers were synthesized by IDT DNA technology (Coralville, IA, USA). Power SYBR green PCR Master Mix (\#4367659, Life Technology) was used for qPCR reactions on $156 \mathrm{nM}$ final primer concentrations and $7 \mathrm{ng} \mathrm{cDNA}$. The reaction was performed on a Bio-Rad (Hercules, CA) CFX96 thermal cycler with the following temperature program: $95^{\circ} \mathrm{C}, 10 \mathrm{~min}, 40$ cycles $\left(60^{\circ} \mathrm{C}, 15 \mathrm{~s} \rightarrow 72^{\circ} \mathrm{C}, 1 \mathrm{~min}\right)$ then melting curve program $\left(95^{\circ} \mathrm{C}, 10 \mathrm{~s}\right.$, then $60^{\circ} \mathrm{C}$ to $95^{\circ} \mathrm{C}, 0.5^{\circ} \mathrm{C}$ increment). The efficiencies of all primers were verified to be between 95 and $105 \%$, using cDNA dilutions spanning 5 magnitudes. The relative amount of RNA was calculated in reference to $\beta$-actin using the $2^{-\Delta \Delta C T}$ method.

2.7. Statistical Analysis. The significance of the differences between nonpaired groups was assessed by Student's unpaired $t$-test with significance defined as a $P<0.05$. Paired, balanced comparisons were performed for Western blot results on a per-dam basis, comparing the mean values between uterine horns by paired $t$-test. The statistical significance of differences involving unbalanced paired comparisons were assessed by resampling as described [17].

\section{Results}

3.1. Induction of Fetal Hyperglycemia. Pregnant dams were infused with $4 \mathrm{mg} / \mathrm{min}$ dextrose such that hyperglycemia exposure occurred in the left but not right uterine horn blood supply. The infusion was initiated on gestational day 19 and continued through the conclusion of the study on gestational day 20. Maternal blood glucose remained euglycemic during the infusion with a small but significant increase in maternal glycemia by the conclusion of the infusion as compared to preinfusion (Figure 1(a)). Fetuses were examined at 24 hours of infusion. At this time, blood glucose was lower in the right-sided fetuses $(57 \pm 8 \mathrm{mg} / \mathrm{dL})$ than in maternal blood $(102 \pm 4)$. These right-sided fetal blood glucose levels would be considered hypoglycemic by postnatal standards but are considered euglycemic for late gestational fetal rats which typically have mean blood glucose levels near $50 \mathrm{mg} / \mathrm{dL}$ [31$34]$. By contrast, left-sided fetuses had $128 \pm 58 \%$ higher glucoses $(131 \pm 33 \mathrm{mg} / \mathrm{dL})$ than right-sided fetuses (Figure 1(b)). Fetal insulin was increased $125 \pm 58 \%$ among left as compared to right-sided fetuses (Figure 1(c)). Maternal blood glucose, per dam, is shown versus fetal blood glucose and weight in Tables 2 and 3 , respectively.

3.2. Cardiac Overgrowth. The primary outcome of interest was whether the 24 hours of hyperglycemia among leftsided fetuses was sufficient to alter the cardiac septal width as compared to the right-sided (euglycemic) fetuses. Thus the diastolic interventricular cardiac septum was measured by echocardiography in exteriorized fetuses on gestational day 20 after 24 hours $4 \mathrm{mg} / \mathrm{min}$ dextrose infusion. The septal thickness was $22.8 \pm 3.2 \%$ greater among left-sided (hyperglycemia exposed, $0.592 \pm 0.016 \mathrm{~mm}$ ) fetuses as compared to that of right-sided (control, $0.482 \pm 0.016 \mathrm{~mm}$ ) fetuses (Figure 2(a)). Likewise, septal thickness normalized to fetal weight was greater among left-sided fetuses $(0.19 \pm 0.02 \mathrm{~mm} / \mathrm{g})$ compared to right-sided fetuses $(0.11 \pm$ $0.01 \mathrm{~mm} / \mathrm{g}$ ) (Figure 2(b)), indicating the septal overgrowth exceeded general body growth. Microscopic sections from left-sided fetuses exhibited relative ventricular and septal wall thickness such that the ventricular cavity space was 


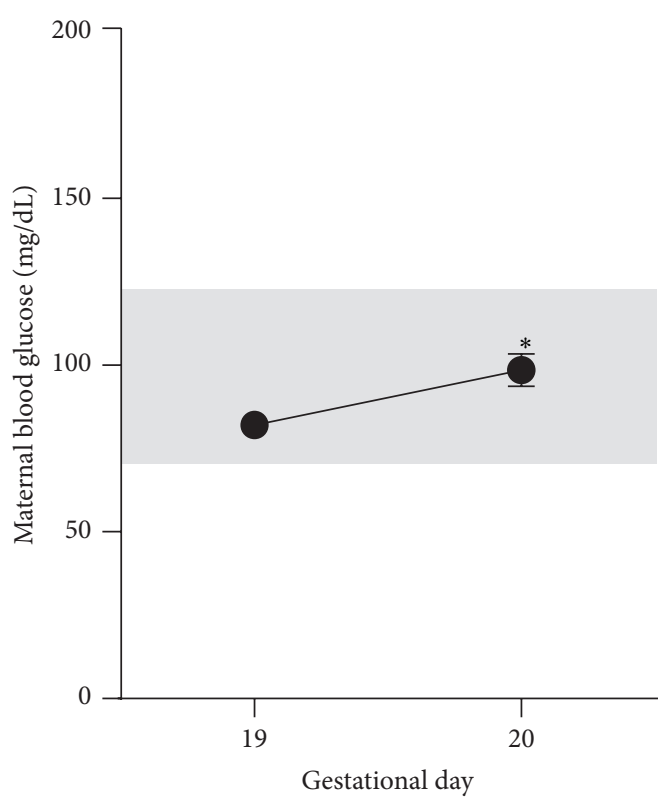

(a)

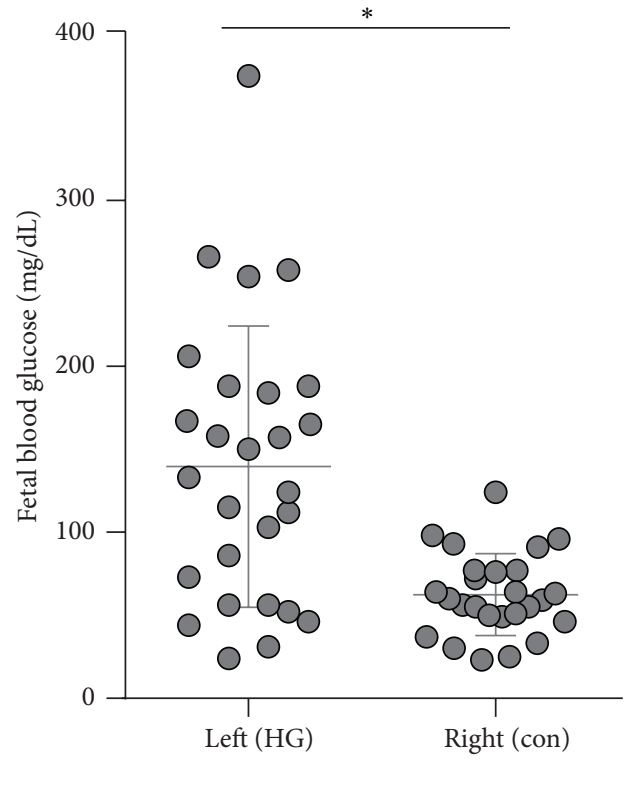

(b)

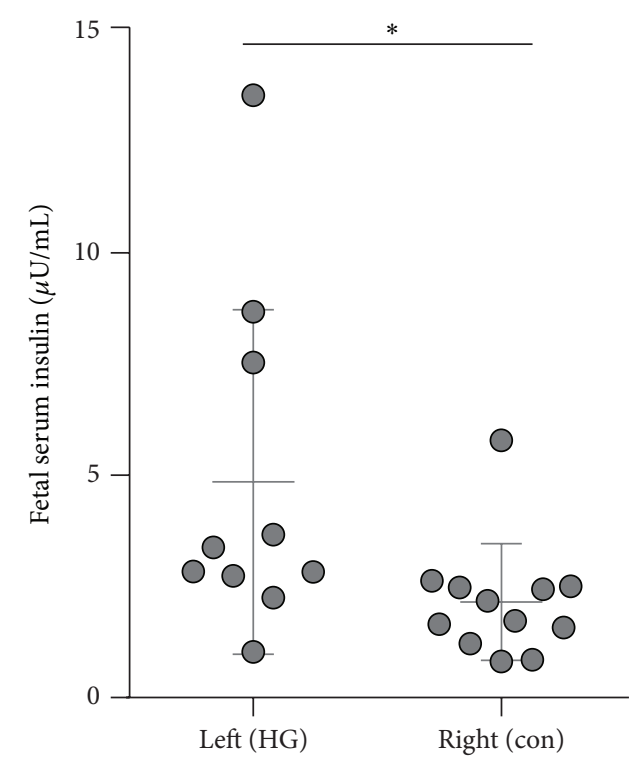

(c)

FIGURE 1: Maternal and fetal glucose and insulin levels. Glucose was infused into the left uterine artery from gestational day 19 until day 20. (a) Maternal blood glucose was measured in the nonfasted state just prior to infusion on gestational day 19 and just before the conclusion of the infusion on gestational day 20 . The euglycemic range (70-130) is highlighted on the figure in grey. ${ }^{*} P<0.001$ by paired $t$-test, $N=10$ dams. (b) Fetal blood glucose was measured just before the conclusion of the infusion for right-sided (i.e., control, "con") and left-sided (i.e., hyperglycemia infused, "HG") fetuses. ${ }^{*} P=0.05$ by paired $t$-test, $N=5$ dams, $26-27$ fetuses. (c) Fetal serum insulin just before the conclusion of the infusion. ${ }^{*} P<0.05$ by $t$-test, $N=4$ dams, $10-12$ fetuses.

reduced and often not even visually evident, as compared to that of right-sided fetuses (Figure 2(c)). Total cardiac weight did not differ between left- and right-sided fetuses $(P=$ ns, $N=4$ dams, 9-33 fetuses). We assessed whether the increased myocardial septal thickness was associated with altered myocardial proliferation by determining which nuclei expressed Ki-67, a nuclear protein present during active portions of the cell cycle but absent from cells resting in the resting $\left(G_{0}\right)$ phase. Interestingly, the percent of nuclei positive for Ki67 was $51 \pm 18 \%$ higher in the septal myocardium of leftsided compared to right-sided fetuses (Figures 2(d) and 2(e)).

\subsection{Impact of Fetal Hyperglycemia on Glucose Anabolism-} Related Gene Expression. The above results show that hyperglycemic exposure is sufficient to induce fetal cardiac septal overgrowth, suggesting the possibility that hyperglycemia 
TABLE 2: Per dam maternal and fetal blood glucoses.

\begin{tabular}{lccc}
\hline \multicolumn{3}{c}{ Maternal blood glucose (mg/dL) } & $\begin{array}{c}\text { a Fetal blood glucose }(\mathrm{mg} / \mathrm{dL}) \\
(\text { mean } \pm \text { SEM) }\end{array}$ \\
${ }^{\mathrm{b}}$ GD19 & GD20 & Left & Right \\
\hline 98 & 124 & $244 \pm 132$ & 56 \\
78 & 97 & $173 \pm 15$ & 46 \\
76 & 80 & $28 \pm 3$ & $37 \pm 5$ \\
97 & 112 & $126 \pm 24$ & $75 \pm 4$ \\
88 & 113 & $168 \pm 18$ & $74 \pm 12$ \\
\hline
\end{tabular}

Each row represents a separate dam. Fetal blood glucose levels without a SEM are from a single fetus. ${ }^{a}$ Measured on gestational day $20 .{ }^{b} \mathrm{GD}$, gestational day.

might directly induce fetal myocardial growth. Glucose can generate cellular growth via the Warburg effect which increases the anaerobic flux of glucose carbons into anabolic pathways. Since maternal diabetes promotes fetal Warburg conditions by both increasing glucose levels and reducing fetal oxygen tension, we wondered whether a Warburg-like shift in gene expression might contribute to the cardiac overgrowth observed in our model. We thus tested the expression of 8 genes known to be induced in the Warburg effect (Table 1). The expression of all these genes was unchanged or even reduced in hyperglycemia exposed hearts (Table 4). These results suggest that a shift to Warburg-like metabolism is not responsible for the fetal cardiac overgrowth induced by hyperglycemic exposure.

3.4. Effect of Hyperglycemia on the Mitogenic Signaling Factors. We investigated the expression levels and activation of intracellular kinases implicated in cardiac overgrowth in the hyperglycemia exposed fetal heart. Hyperglycemic exposure had no effect on the phosphorylation of AKT, ERK, or P38. By contrast, there was a $2.5 \pm 0.4$ fold induction of phosphorylated JNK levels as a result of hyperglycemic exposure (Figures 3(a) and 3(b)). As confirmation of this result, we studied the effect of longer duration of hyperglycemic exposure in a separate cohort of dams infused for 48 hours on gestational days 18-20. JNK signaling activation was maintained at least through 48 hours of hyperglycemic exposure (Figure 3(c)). We also investigated the expression of IGF-1, a paracrine factor highly implicated in cardiac overgrowth [35], and Glut4, a major glucose transporter in the heart and modulator of cardiac growth [36]. Interestingly, Glut4 expression was mildly increased by hyperglycemia exposure, whereas IGF-1 levels were unchanged (Figure 3).

\section{Discussion}

Although the association of maternal diabetes and newborn cardiac overgrowth has been recognized since the 1940s [37], the causative relationship between maternal hyperglycemia and cardiac overgrowth has been unclear. This uncertainty is highlighted by numerous epidemiologic studies that find no relationship between the degree of maternal hyperglycemia and cardiac overgrowth $[2,4,5,9,10]$. Herein, we directly tested the hypothesis that maternal hyperglycemia is sufficient to induce fetal cardiac septal overgrowth by using a recently developed model whereby glucose is infused into the left uterine artery [16]. This approach isolates the effects of hyperglycemia to just the fetuses developing in left uterine horn, with the fetuses in the right horn serving as controls. The cardiac septal width of the hyperglycemia exposed fetuses was significantly greater than the control fetuses, showing that indeed maternal hyperglycemia alone is sufficient to induce cardiac septal overgrowth. This result is consistent with those epidemiologic studies that do find that the degree of maternal hyperglycemia predicts newborn cardiac overgrowth $[3,8]$.

It is thus an open question as to why multiple studies have not observed a relationship between maternal hyperglycemia and newborn cardiac overgrowth. One possibility that has been forwarded is that other derangements of diabetic pregnancy, such as ketonemia [38] or hyperlipidemia [39], contribute to the fetal septal overgrowth. The studies reported in this paper are aimed at glucose alone and thus do not address these alternative possibilities. It thus remains possible that nonglycemic aspects of diabetic pregnancy may contribute to septal overgrowth, in concert with or independent of hyperglycemia. The rapidity by which hyperglycemia induced fetal cardiac overgrowth was surprising. This informative result however suggests a possible reason for the apparent lack of association between markers of chronic maternal hyperglycemia and fetal septal overgrowth. Namely, it suggests that only transient episodes of hyperglycemia are sufficient to induce septal overgrowth and that chronic hyperglycemia may not be required. Though this finding could have clinical implications in terms of avoiding even transient spikes of hyperglycemia during pregnancy, we consider that application of this implication to the clinical realm is currently premature and requires additional translational studies. For example, it is not clear how brief of a hyperglycemic exposure might be required to induce human fetal cardiac overgrowth. Using our model system it is possible, albeit labor intensive, to define the minimal length of exposure required to induce cardiac overgrowth in the fetal rat. However, there is no clear approach to correlate such a length to the human fetal situation.

The mechanisms underlying diabetes-induced fetal cardiac overgrowth are unclear. One interesting mechanistic possibility is that glucose directly promotes cardiac overgrowth. A well described mechanism by which glucose directly enhances cellular growth is the Warburg effect, which supports the anabolic state of malignant cells. Our results indicate that hyperglycemic exposure does not induce a Warburg-like shift in gene expression in the fetal myocardium. Because the fetal environment is hypoxic, anabolic, and glycolytic at baseline $[27,28]$, it is possible that the fetal myocardium may already be in a Warburglike state at baseline and thus susceptible to glucose driven overgrowth. Our experiments cannot exclude this possibility. Another important mechanism in cardiac overgrowth is activation of growth/stress signaling kinases including AKT and MAP-kinases JNK, ERK, and p38 [18-21]. Of these, we found that cardiac JNK is activated by fetal hyperglycemic 
TABLE 3: Per dam maternal blood glucose and fetal weight.

\begin{tabular}{|c|c|c|c|}
\hline \multicolumn{2}{|c|}{ Maternal blood glucose (mg/dL) } & \multicolumn{2}{|c|}{${ }^{\mathrm{a}}$ Fetal weight $(\mathrm{g})($ mean \pm SEM $)$} \\
\hline${ }^{\mathrm{b}} \mathrm{GD} 19$ & GD20 & Left & Right \\
\hline 78 & 94 & $3.748 \pm 0.082$ & $3.880 \pm 0.065$ \\
\hline 74 & 103 & $3.165 \pm 0.185$ & $3.650 \pm 0.089$ \\
\hline 75 & 115 & $2.890 \pm 0.239$ & $3.128 \pm 0.146$ \\
\hline 81 & 110 & $2.810 \pm 0.070$ & $3.622 \pm 0.058$ \\
\hline 88 & 113 & 3.510 & $3.667 \pm 0.081$ \\
\hline
\end{tabular}

Each row represents a separate dam. ${ }^{\mathrm{a}}$ Measured on gestational day $20 .{ }^{\mathrm{b}} \mathrm{GD}$, gestational day.

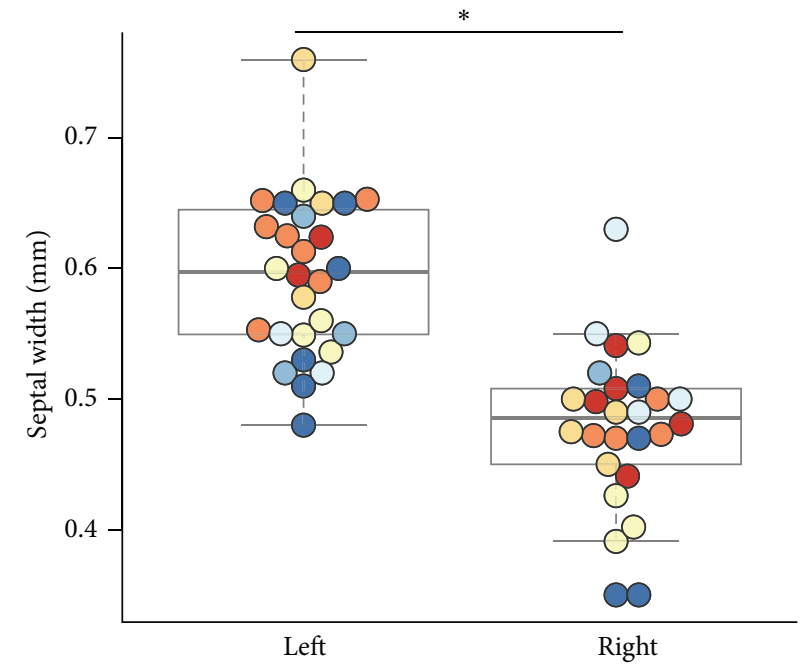

(a)

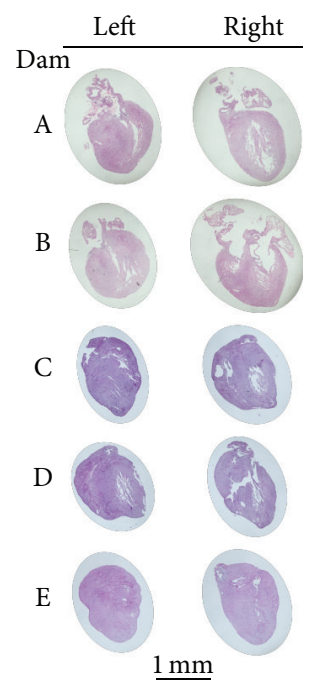

(c)

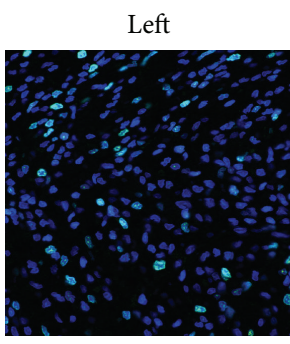

Green $=$ ki67

Blue $=$ TOPRO3

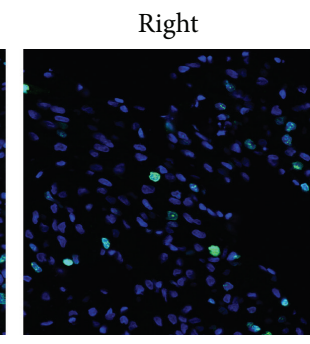

(d)

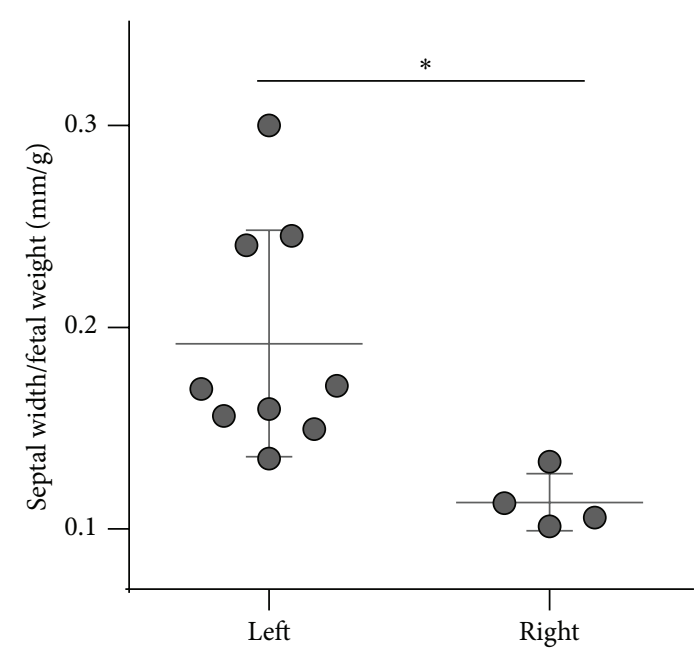

(b)

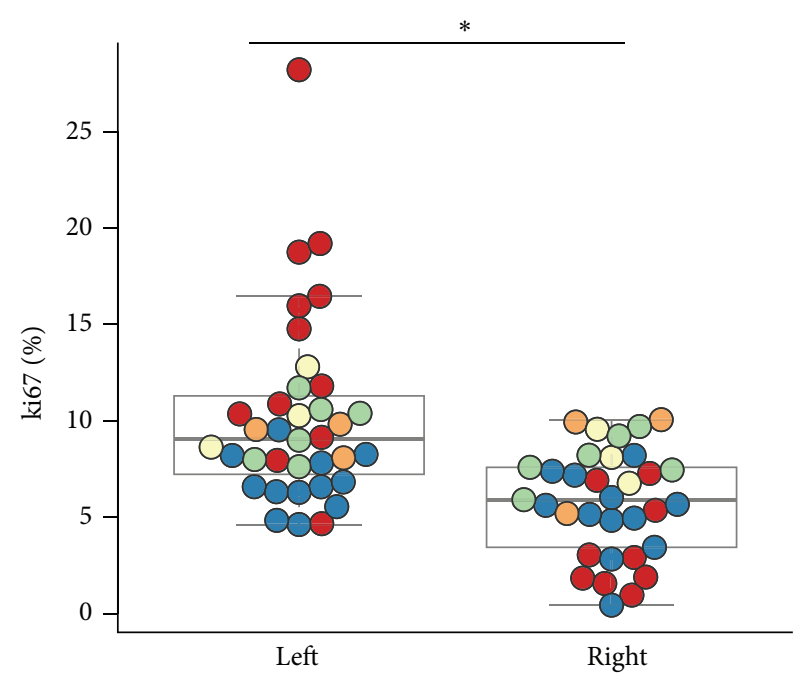

(e)

FIGURE 2: Cardiac septal width and myocardial proliferation. (a) Fetal cardiac septal width, as measured by echocardiograph, among left-sided (i.e., hyperglycemia infused) and right-sided (control) fetuses. Differing colors indicate different dams. ${ }^{*} P<0.0005$ by resampling, $N=7$ dams, $N=26$, 28 fetuses per side. (b) The ratio of cardiac septal width to fetal weight. ${ }^{*} P<0.05$ by $t$-test, $N=4,9$ fetuses per side, 2 dams. (c) Hematoxylin and eosin stained fetal hearts from the left (hyperglycemia) and right (control) uterine horns. (d) Myocardial proliferation as evidenced by immunofluorescence detection of ki67 (green) and DAPI (blue). Representative images from the interventricular septal region are shown. (e) The percentage of ki67 positivity among nuclei in the fetal myocardium. ${ }^{*} P<0.0005$ by resampling, $N=5$ dams, $>500$ nuclei from 3 myocardial regions (left, right, septal ventriculum) analyzed per fetus. $N=33,36$ fetuses per side. 


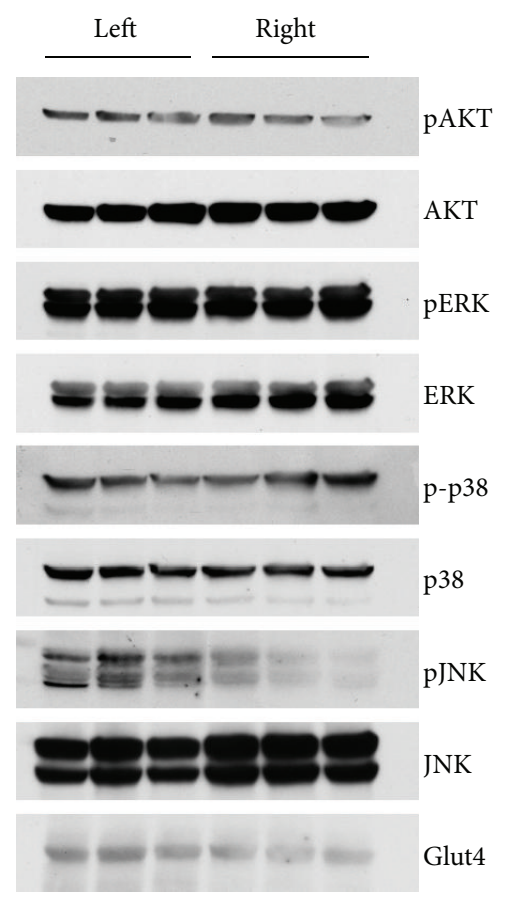

(a)

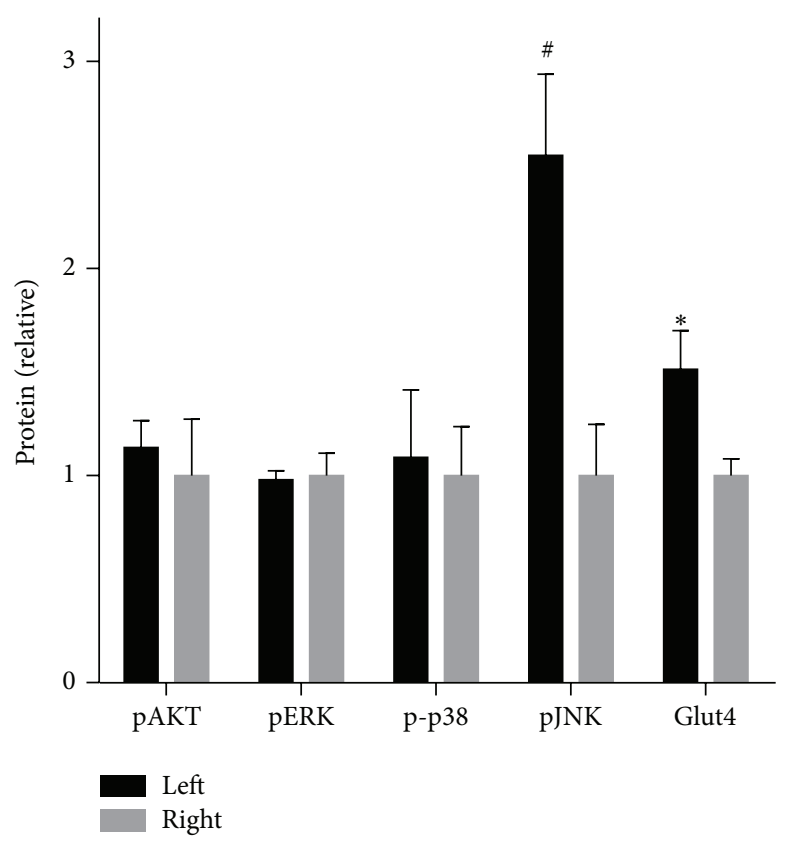

(b)

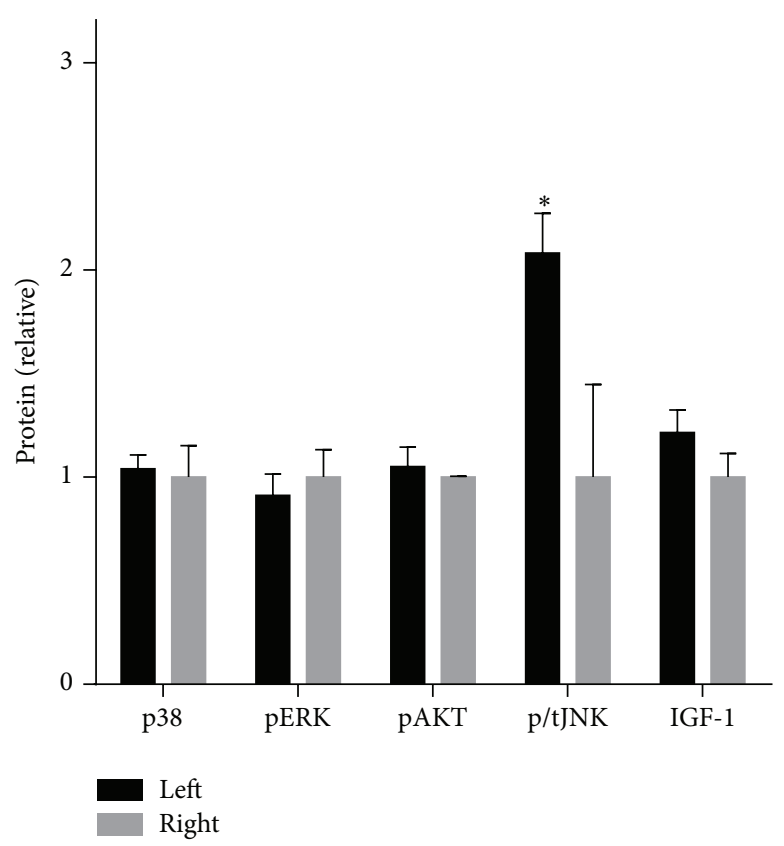

(c)

Figure 3: Myocardial protein levels in the heart from left (hyperglycemia exposed) and right (control) fetuses. (a) Western blot of phosphokinase and total kinase levels (AKT, ERK, p38, and JNK) and glucose transporter 4 (Glut4). (b) Quantitation of Western blot results. $N=4$ dams, 6 fetuses per side. ${ }^{*} P<0.01,{ }^{*} P<0.005$. (c) Quantitation of Western blot results. $N=1,2,3,2,3$ dams and $4,8,12,3,12$ fetuses/side for phospho-p38, phospho-ERK, insulin stimulated phospho-AKT, phospho-JNK normalized to total, and IGF-1, respectively. ${ }^{*} P<0.01$. (a-b) Dams infused with left uterine artery hyperglycemia on gestational days 19-20. (c) Dams infused with left uterine artery hyperglycemia on gestational days 18-20. 
TABLE 4: Relative expression of genes related to Warburg metabolism.

\begin{tabular}{|c|c|c|c|c|c|}
\hline \multirow{2}{*}{ Gene } & \multicolumn{2}{|c|}{ Right } & \multicolumn{2}{|c|}{ Left } & \multirow{2}{*}{$P$} \\
\hline & Ave \pm SEM & $N$ & Ave \pm SEM & $N$ & \\
\hline Tigar & $1.00 \pm 0.06$ & 5 & $1.07 \pm 0.08$ & 6 & 0.47 \\
\hline$P k m 2$ & $1.00 \pm 0.10$ & 5 & $1.03 \pm 0.07$ & 6 & 0.80 \\
\hline Gpt2 & $1.00 \pm 0.05$ & 5 & $1.07 \pm 0.05$ & 6 & 0.35 \\
\hline$H k 2$ & $1.00 \pm 0.08$ & 5 & $0.74 \pm 0.11$ & 6 & 0.10 \\
\hline$P d k 1$ & $1.00 \pm 0.08$ & 5 & $0.89 \pm 0.08$ & 6 & 0.39 \\
\hline P53 & $1.00 \pm 0.11$ & 5 & $1.01 \pm 0.07$ & 6 & 0.93 \\
\hline Ldha & $1.00 \pm 0.07$ & 5 & $1.06 \pm 0.05$ & 6 & 0.49 \\
\hline Glut1 & $1.00 \pm 0.12$ & 5 & $1.02 \pm 0.09$ & 6 & 0.92 \\
\hline
\end{tabular}

Expression of genes related to Warburg metabolism. Relative mRNA levels in fetal myocardium were determined by real time PCR. $P$ is nonsignificant for all genes tested, $N=5-6$ fetuses per side, 2 dams. Gene abbreviations are identical to those in Table 1.

exposure. JNK has been implicated in the pathogenesis of cardiac hypertrophy during postnatal life $[40,41]$ including in response to diabetes [19]. On the other hand, JNK has been implicated in contributing to the resolution of cardiac overgrowth [42, 43]. It is thus unclear which of these two roles, detrimental or adaptive, is played by JNK activation in our model. It is possible that studies employing JNK inhibitors could aid in distinguishing between the two opposing possibilities. We found increased levels of Glut4 in hyperglycemia exposed fetal heart, whereas profound deficiency of Glut 4 is described to induce cardiac hypertrophy [36]. Little is known about how diabetes affects the expression of Glut 4 in fetal tissues, although increased [44] or decreased [45] Glut4 expression has been found in human placenta from diabetic pregnancies. Although IGF-1 levels are increased in fetal serum in diabetic pregnancy [46] and IGF-1 is considered to be a major factor promoting cardiac growth, its expression was not altered in hyperglycemia hearts in our model. There is very little published about the impact of maternal diabetes on the expression of Glut 4 and IGF-I in the fetal heart, and thus it is not possible to speculate how maternal diabetes might differ from hyperglycemia alone in this regard. The majority of prior work on cellular signaling during cardiac overgrowth has been conducted in the adult heart or in cultured neonatal cardiac myocytes. It is possible that the signaling events that underlie fetal cardiac overgrowth in vivo differ from those in play during postnatal life.

The fetal heart is an insulin-responsive tissue $[47,48]$ and it has long been hypothesized that elevated fetal insulin induced by maternal diabetes may contribute to fetal cardiac overgrowth [1]. Insulin signaling contributes to both embryonic and postnatal cardiac growth [49]. Furthermore, excess insulin signaling contributes to cardiac hypertrophy and dysfunction [50]. In fact, newborns with primary hyperinsulinism exhibit hypertrophic cardiomyopathy [51]. Fetal insulin levels during exposure to maternal diabetes are often elevated due to the fetal beta-cell response to hyperglycemia. For this reason, it has been difficult to determine whether it is fetal hyperglycemia, fetal hyperinsulinemia, or both that drives cardiac overgrowth in response to maternal diabetes. Our present results likewise do not distinguish between these possibilities since both glucose and insulin are elevated in the left uterine horn fetuses. Thus, it is possible that insulin may be a key factor in the observed cardiac septal overgrowth.

Diabetes-induced fetal cardiac overgrowth has long been described as "hypertrophy" [37], dating prior to the advent of modern techniques that measure cell replication. Myocardial growth during fetal development occurs primarily through cardiac myocyte proliferation, whereas postnatal heart growth occurs through hypertrophy [52]. Our current results indicate that the heart septal overgrowth is accompanied by increased replication, suggesting cardiac hyperplasia. This result is consistent with recent studies also demonstrating fetal cardiac hyperplasia in diabetic pregnant mice [53]. In the present paper we have favored the term "overgrowth" rather than the classic term "hypertrophy" to indicate the uncertainty as to whether the septal enlargement involves just hyperplasia or also hypertrophy. This is an important distinction since the mechanisms that underlie cardiac hyperplasia may be different from those that produce hypertrophy. Our recent findings that cell cycle genes are perturbed in a different model of prenatal cardiac overgrowth speak to this possibility [54].

Our study occurred during late gestation in the fetal rat. At this stage, the heart is structurally fully developed and has been compared to human midgestation based on cardiac morphometry [55] or second-half gestation based on myofibril content [56]. Importantly, the ability of hyperglycemia to promote cardiac overgrowth may be limited to distinct developmental cardiac stages.

Our experimental model system has advantages and disadvantages compared to other in vivo models of diabetic pregnancy. One advantage is the ability to isolate the effects of maternal hyperglycemia alone. Another advantage is complete temporal control over the timing of hyperglycemic exposure. Disadvantages to our model include its labor intensive and challenging surgical nature. Additionally, the total hyperglycemic exposure and excess glucose received per fetus cannot be directly measured. A rough estimate can be extrapolated from glucose tracer experiments [16] showing that each left-sided rat fetus receives approximately $2.3 \%$ of the infused glucose tracer, which summed over 24 hours of infusion at $4 \mathrm{mg} / \mathrm{min}$ amounts to $\sim 130 \mathrm{mg}$ excess glucose per fetus. Although our model system isolates 
the local maternal effects of hyperglycemia on the fetus, this is not equivalent to producing hyperglycemia alone in the fetus as there will be a number of fetal responses and even local maternal responses to the hyperglycemia. Presumably the hyperglycemia-induced cardiac overgrowth is reversible, just as in human and rodent fetal diabetic cardiac overgrowth, although this was not examined in our model. In human diabetic pregnancy, blood glucose levels often fluctuate considerably, and it is thus possible but not tested that pulsatile infusion of glucose might also result in cardiac overgrowth. Along these lines, an interesting experimental possibility introduced by our model system is to study the acute signaling events that occur in response to the sudden appearance or removal of hyperglycemic exposure. Such studies may help better understand the short and long term effects of fetal hyperglycemic exposure.

In summary, our results demonstrate that maternal hyperglycemia is sufficient to rapidly induce cardiac septal overgrowth. Only transient hyperglycemic exposure is required, possibly explaining why markers of chronic hyperglycemia often do not predict the degree of overgrowth. Increased insulin levels and JNK activation may contribute to this process.

\section{Conflict of Interests}

The authors declare that there is no conflict of interests regarding the publication of this paper.

\section{Acknowledgments}

This work was supported by a University of Iowa Internal Funding Initiatives Award, a pilot grant from the Fraternal Order of Eagles Diabetes Research Center, by R24 DK96518 and by R01 DK097820.

\section{References}

[1] H. P. Gutgesell, M. E. Speer, and H. S. Rosenberg, "Characterization of the cardiomyopathy in infants of diabetic mothers," Circulation, vol. 61, no. 2, pp. 441-450, 1980.

[2] J. Åman, U. Hansson, I. Östlund, K. Wall, and B. Persson, "Increased fat mass and cardiac septal hypertrophy in newborn infants of mothers with well-controlled diabetes during pregnancy," Neonatology, vol. 100, no. 2, pp. 147-154, 2011.

[3] M. J. Cooper, M. A. Enderlein, H. Tarnoff, and C. L. Rogé, "Asymmetric septal hypertrophy in infants of diabetic mothers: fetal echocardiography and the impact of maternal diabetic control," American Journal of Diseases of Children, vol. 146, no. 2, pp. 226-229, 1992.

[4] P. Q. Sheehan, T. W. Rowland, B. L. Shah, V. J. McGravey, and E. O. Reiter, "Maternal diabetic control and hypertrophic cardiomyopathy in infants of diabetic mothers," Clinical Pediatrics, vol. 25, no. 5, pp. 266-271, 1986.

[5] M. M. Vela-Huerta, A. Vargas-Origel, and A. Olvera-López, "Asymmetrical septal hypertrophy in newborn infants of diabetic mothers," American Journal of Perinatology, vol. 17, no. 2, pp. 89-94, 2000.
[6] R. M. Abu-Sulaiman and B. Subaih, "Congenital heart disease in infants of diabetic mothers: echocardiographic study," Pediatric Cardiology, vol. 25, no. 2, pp. 137-140, 2004.

[7] M. Mongiovi, V. Fesslova, G. Fazio, G. Barbaro, and S. Pipitone, "Diagnosis and prognosis of fetal cardiomyopathies: a review," Current Pharmaceutical Design, vol. 16, no. 26, pp. 2929-2934, 2010.

[8] M. M. El-Ganzoury, S. A. El-Masry, R. A. El-Farrash, M. Anwar, and R. Z. Abd Ellatife, "Infants of diabetic mothers: echocardiographic measurements and cord blood IGF-I and IGFBP-1," Pediatric Diabetes, vol. 13, no. 2, pp. 189-196, 2012.

[9] Y. Ren, Q. Zhou, Y. Yan, C. Chu, Y. Gui, and X. Li, "Characterization of fetal cardiac structure and function detected by echocardiography in women with normal pregnancy and gestational diabetes mellitus," Prenatal Diagnosis, vol. 31, no. 5, pp. 459-465, 2011.

[10] J. M. Alsweiler, J. E. Harding, and F. H. Bloomfield, "Tight glycemic control with insulin in hyperglycemic preterm babies: a randomized controlled trial," Pediatrics, vol. 129, no. 4, pp. 639-647, 2012.

[11] H. S. Weber, J. J. Botti, and B. G. Baylen, "Sequential longitudinal evaluation of cardiac growth and ventricular diastolic filling in fetuses of well controlled diabetic mothers," Pediatric Cardiology, vol. 15, no. 4, pp. 184-189, 1994.

[12] J. A. Gandhi, X. Y. Zhang, and J. E. Maidman, "Fetal cardiac hypertrophy and cardiac function in diabetic pregnancies," American Journal of Obstetrics \& Gynecology, vol. 173, no. 4, pp. 1132-1136, 1995.

[13] H. S. Weber, J. A. Copel, E. A. Reece, J. Green, and C. S. Kleinman, "Cardiac growth in fetuses of diabetic mothers with good metabolic control," The Journal of Pediatrics, vol. 118, no. 1, pp. 103-107, 1991.

[14] R. Oberhoffer, J. Högel, F. Stoz, E. Kohne, and D. Lang, “Cardiac and extracardiac complications in infants of diabetic mothers and their relation to parameters of carbohydrate metaholism," European Journal of Pediatrics, vol. 156, no. 4, pp. 262-265, 1997.

[15] A. R. Hayati, F. C. Cheah, J. F. Yong, A. E. Tan, and W. M. Norizah, "The role of serum insulin-like growth factor I (IGF-I) in neonatal outcome," Journal of Clinical Pathology, vol. 57, no. 12, pp. 1299-1301, 2004.

[16] J. Yao, C. Wang, S. A. Walsh et al., "Localized fetomaternal hyperglycemia: spatial and kinetic definition by positron emission tomography," PLoS ONE, vol. 5, no. 8, Article ID e12027, 2010.

[17] M. L. Baack, C. Wang, S. Hu, J. L. Segar, and A. W. Norris, "Hyperglycemia induces embryopathy, even in the absence of systemic maternal diabetes: an in vivo test of the fuel mediated teratogenesis hypothesis," Reproductive Toxicology, vol. 46, pp. 129-136, 2014.

[18] T. Yamazaki, I. Komuro, I. Shiojima, and Y. Yazaki, "Angiotensin II mediates mechanical stress-induced cardiac hypertrophy," Diabetes Research and Clinical Practice, vol. 30, supplement, pp. 107-111, 1996.

[19] E. Shen, X. Diao, X. Wang, R. Chen, and B. Hu, "MicroRNAs involved in the mitogen-activated protein kinase cascades pathway during glucose-induced cardiomyocyte hypertrophy," The American Journal of Pathology, vol. 179, no. 2, pp. 639-650, 2011.

[20] Z. Chang, Q. Zhang, Q. Feng et al., "Deletion of Aktl causes heart defects and abnormal cardiomyocyte proliferation," Developmental Biology, vol. 347, no. 2, pp. 384-391, 2010. 
[21] O. F. Bueno, L. J. De Windt, K. M. Tymitz et al., "The MEK1-ERK1/2 signaling pathway promotes compensated cardiac hypertrophy in transgenic mice," The EMBO Journal, vol. 19, no. 23, pp. 6341-6350, 2000.

[22] A. J. Levine and A. M. Puzio-Kuter, "The control of the metabolic switch in cancers by oncogenes and tumor suppressor genes," Science, vol. 330, no. 6009, pp. 1340-1344, 2010.

[23] H. R. Christofk, M. G. V. Heiden, M. H. Harris et al., "The M2 splice isoform of pyruvate kinase is important for cancer metabolism and tumour growth," Nature, vol. 452, no. 7184, pp. 230-233, 2008.

[24] M. G. Vander Heiden, J. W. Locasale, K. D. Swanson et al., "Evidence for an alternative glycolytic pathway in rapidly proliferating cells," Science, vol. 329, no. 5998, pp. 1492-1499, 2010.

[25] B. K. Redel, A. N. Brown, L. D. Spate, K. M. Whitworth, J. A. Green, and R. S. Prather, "Glycolysis in preimplantation development is partially controlled by the Warburg effect," Molecular Reproduction and Development, vol. 79, no. 4, pp. 262-271, 2012.

[26] W. Ma, H. J. Sung, J. Y. Park, S. Matoba, and P. M. Hwang, "A pivotal role for p53: balancing aerobic respiration and glycolysis," Journal of Bioenergetics and Biomembranes, vol. 39, no. 3, pp. 243-246, 2007.

[27] J. L. Nold and M. K. Georgieff, "Infants of diabetic mothers," Pediatric Clinics of North America, vol. 51, no. 3, pp. 619-637, 2004.

[28] A. F. Philipps, P. J. Porte, S. Stabinsky, T. S. Rosenkrantz, and J. R. Raye, "Effects of chronic fetal hyperglycemia upon oxygen consumption in the ovine uterus and conceptus," Journal of Clinical Investigation, vol. 74, no. 1, pp. 279-286, 1984.

[29] A. Untergasser, H. Nijveen, X. Rao, T. Bisseling, R. Geurts, and J. A. M. Leunissen, "Primer3Plus, an enhanced web interface to Primer3," Nucleic Acids Research, vol. 35, no. 2, pp. W71-W74, 2007.

[30] M. Zuker, "Mfold web server for nucleic acid folding and hybridization prediction," Nucleic Acids Research, vol. 31, no. 13, pp. 3406-3415, 2003.

[31] H. Takei, S. Iizuka, M. Yamamoto, S. Takeda, and K. Arishima, "The herbal medicine Tokishakuyakusan increases fetal blood glucose concentrations and growth hormone levels and improves intrauterine growth retardation induced by $\mathrm{N}$ (omega)-nitro-L-arginine methyl ester," Journal of Pharmacological Sciences, vol. 104, no. 4, pp. 319-328, 2007.

[32] J. Lesage, D. Hahn, M. Léonhardt, B. Blondeau, B. Bréant, and J. P. Dupouy, "Maternal undernutrition during late gestationinduced intrauterine growth restriction in the rat is associated with impaired placental GLUT3 expression, but does not correlate with endogenous corticosterone levels," The Journal of Endocrinology, vol. 174, no. 1, pp. 37-43, 2002.

[33] R. M. Lewis, L. A. James, J. Zhang, C. D. Byrne, and C. N. Hales, "Effects of maternal iron restriction in the rat on hypoxiainduced gene expression and fetal metabolite levels," The British Journal of Nutrition, vol. 85, no. 2, pp. 193-201, 2001.

[34] H. F. Sadiq, U. G. Das, T. F. Tracy, and S. U. Devaskar, "Intrauterine growth restriction differentially regulates perinatal brain and skeletal muscle glucose transporters," Brain Research, vol. 823, no. 1-2, pp. 96-103, 1999.

[35] N. R. Sundaresan, P. Vasudevan, L. Zhong et al., "The sirtuin SIRT6 blocks IGF-Akt signaling and development of cardiac hypertrophy by targeting c-Jun," Nature Medicine, vol. 18, no. 11, pp. 1643-1650, 2012.
[36] S. J. Kaczmarczyk, S. Andrikopoulos, J. Favaloro et al., “Threshold effects of glucose transporter-4 (GLUT4) deficiency on cardiac glucose uptake and development of hypertrophy," Journal of Molecular Endocrinology, vol. 31, no. 3, pp. 449-459, 2003.

[37] H. C. Miller and H. M. Wilson, "Macrosomia, cardiac hypertrophy, erythroblastosis, and hyperplasia of the islands of langerhans in infants born to diabetic mothers," The Journal of Pediatrics, vol. 23, no. 3, pp. 251-266, 1943.

[38] M. Miodovnik, J. P. Lavin, D. J. Harrington, L. Leung, A. E. Seeds, and K. E. Clark, "Cardiovascular and biochemical effects of infusion of beta hydroxybutyrate into the fetal lamb," American Journal of Obstetrics \& Gynecology, vol. 144, no. 5, pp. 594-600, 1982.

[39] C. A. Riquelme, J. A. Magida, B. C. Harrison et al., "Fatty acids identified in the Burmese python promote beneficial cardiac growth," Science, vol. 334, no. 6055, pp. 528-531, 2011.

[40] H. Lou, I. Danelisen, and P. K. Singal, "Involvement of mitogenactivated protein kinases in adriamycin-induced cardiomyopathy," American Journal of Physiology-Heart and Circulatory Physiology, vol. 288, no. 4, pp. H1925-H1930, 2005.

[41] M. T. Ramirez, V. P. Sah, X.-L. Zhao, J. J. Hunter, K. R. Chien, and J. H. Brown, "The MEKK-JNK pathway is stimulated by $\alpha 1-$ adrenergic receptor and Ras activation and is associated with in vitro and in vivo cardiac hypertrophy," The Journal of Biological Chemistry, vol. 272, no. 22, pp. 14057-14061, 1997.

[42] Q. Liang and J. D. Molkentin, "Redefining the roles of p38 and JNK signaling in cardiac hypertrophy: dichotomy between cultured myocytes and animal models," Journal of Molecular and Cellular Cardiology, vol. 35, no. 12, pp. 1385-1394, 2003.

[43] B. E. Reinking, E. W. Wedemeyer, R. M. Weiss, J. L. Segar, and T. D. Scholz, "Cardiomyopathy in offspring of diabetic rats is associated with activation of the MAPK and apoptotic pathways," Cardiovascular Diabetology, vol. 8, article 43, 2009.

[44] M. D. Nitert, H. L. Barrett, M. H. Kubala et al., "Increased placental expression of fibroblast growth factor 21 in gestational diabetes mellitus," The Journal of Clinical Endocrinology and Metabolism, vol. 99, no. 4, pp. E591-E598, 2014.

[45] M. Colomiere, M. Permezel, C. Riley, G. Desoye, and M. Lappas, "Defective insulin signaling in placenta from pregnancies complicated by gestational diabetes mellitus," European Journal of Endocrinology, vol. 160, no. 4, pp. 567-578, 2009.

[46] Z.-C. Luo, A.-M. Nuyt, E. Delvin et al., "Maternal and fetal IGF-I and IGF-II levels, fetal growth, and gestational diabetes," Journal of Clinical Endocrinology and Metabolism, vol. 97, no. 5, pp. 1720-1728, 2012.

[47] C. M. Clark Jr., "The stimulation by insulin of amino acid uptake and protein synthesis in the isolated fetal rat heart," Biology of the Neonate, vol. 19, no. 4, pp. 379-388, 1971.

[48] S. U. Devaskar, K. Szewczyk, and U. P. Devaskar, “The fetal heart insulin receptor responds differently to varying plasma insulin concentrations," Developmental Pharmacology and Therapeutics, vol. 10, no. 3, pp. 153-162, 1987.

[49] L. Bertrand, S. Horman, C. Beauloye, and J.-L. Vanoverschelde, "Insulin signalling in the heart," Cardiovascular Research, vol. 79, no. 2, pp. 238-248, 2008.

[50] I. Shimizu, T. Minamino, H. Toko et al., "Excessive cardiac insulin signaling exacerbates systolic dysfunction induced by pressure overload in rodents," The Journal of Clinical Investigation, vol. 120, no. 5, pp. 1506-1514, 2010.

[51] T. T. Huang, A. Kelly, S. A. Becker, M. S. Cohen, and C. A. Stanley, "Hypertrophic cardiomyopathy in neonates with 
congenital hyperinsulinism," Archives of Disease in Childhood: Fetal and Neonatal Edition, vol. 98, no. 4, pp. F351-F354, 2013.

[52] C. T. Jones and T. P. Rolph, "Metabolism during fetal life: a functional assessment of metabolic development," Physiological Reviews, vol. 65, no. 2, pp. 357-430, 1985.

[53] L. Lehtoranta, O. Vuolteenaho, V. J. Laine et al., "Maternal hyperglycemia leads to fetal cardiac hyperplasia and dysfunction in a rat model," American Journal of PhysiologyEndocrinology and Metabolism, vol. 305, no. 5, pp. E611-E619, 2013.

[54] A. W. Norris, T. M. Bahr, T. D. Scholz, E. S. Peterson, K. A. Volk, and J. L. Segar, "Angiotensin II-induced cardiovascular load regulates cardiac remodeling and related gene expression in late-gestation fetal sheep," Pediatric Research, vol. 75, no. 6, pp. 689-696, 2014.

[55] N. J. Sissman, "Developmental landmarks in cardiac morphogenesis: comparative chronology," The American Journal of Cardiology, vol. 25, no. 2, pp. 141-148, 1970.

[56] S. R. Boerth, D. B. Zimmer, and M. Artman, "Steady-state mRNA levels of the sarcolemmal $\mathrm{Na}^{+}-\mathrm{Ca}^{2+}$ exchanger peak near birth in developing rabbit and rat hearts," Circulation Research, vol. 74, no. 2, pp. 354-359, 1994. 


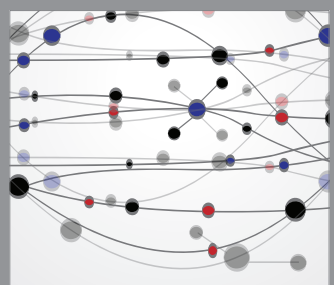

The Scientific World Journal
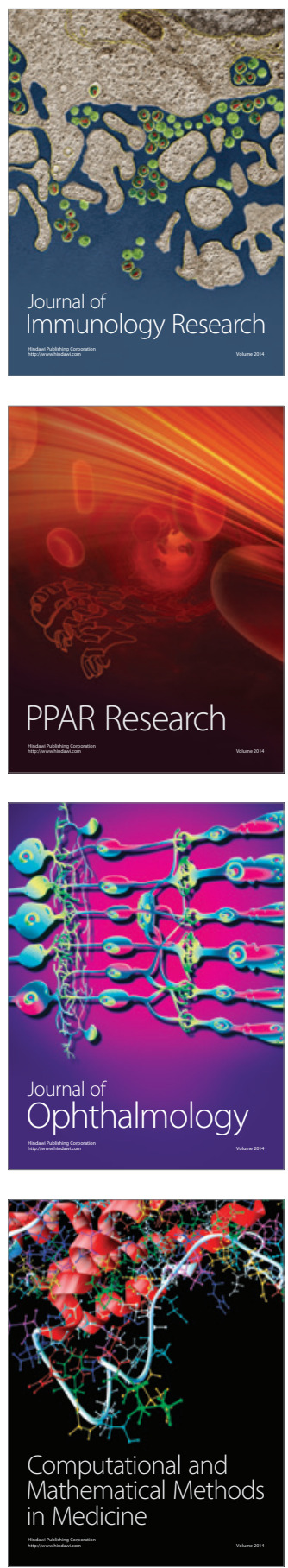

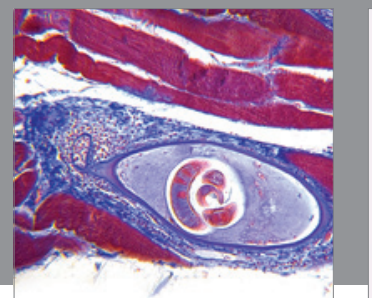

Gastroenterology

Research and Practice
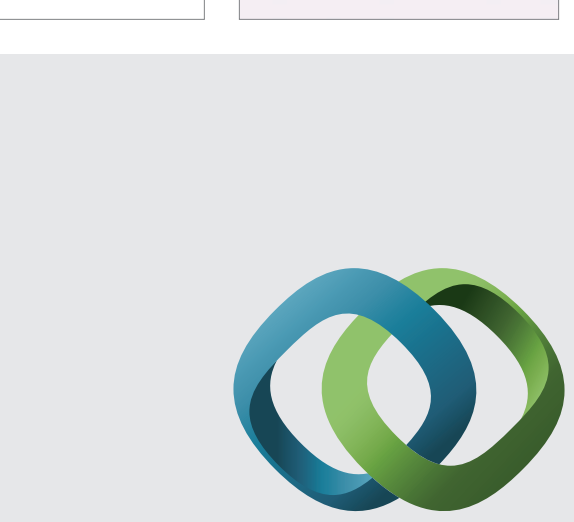

\section{Hindawi}

Submit your manuscripts at

http://www.hindawi.com
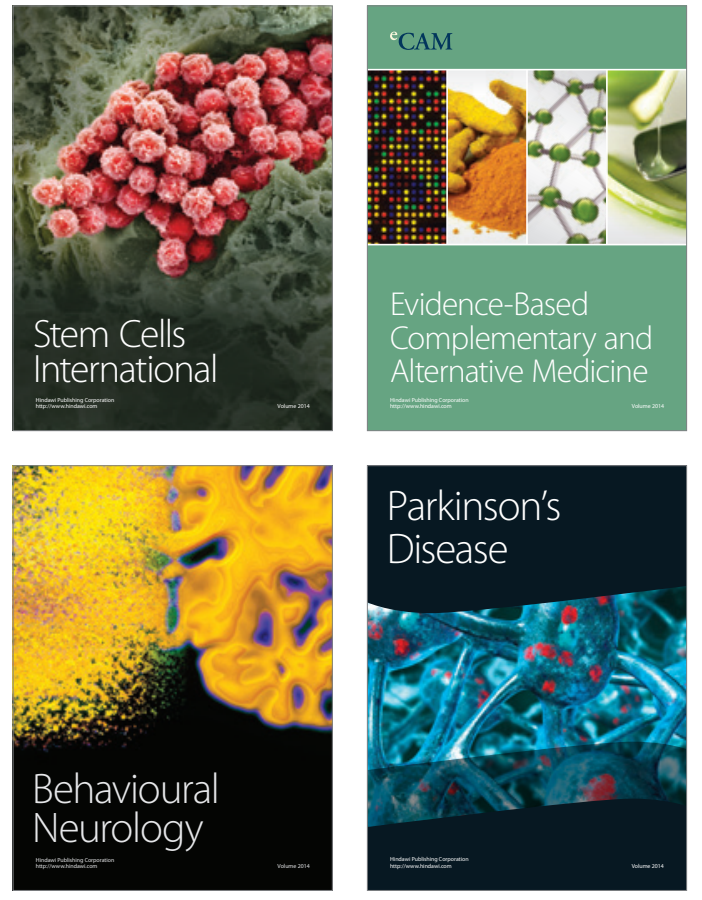
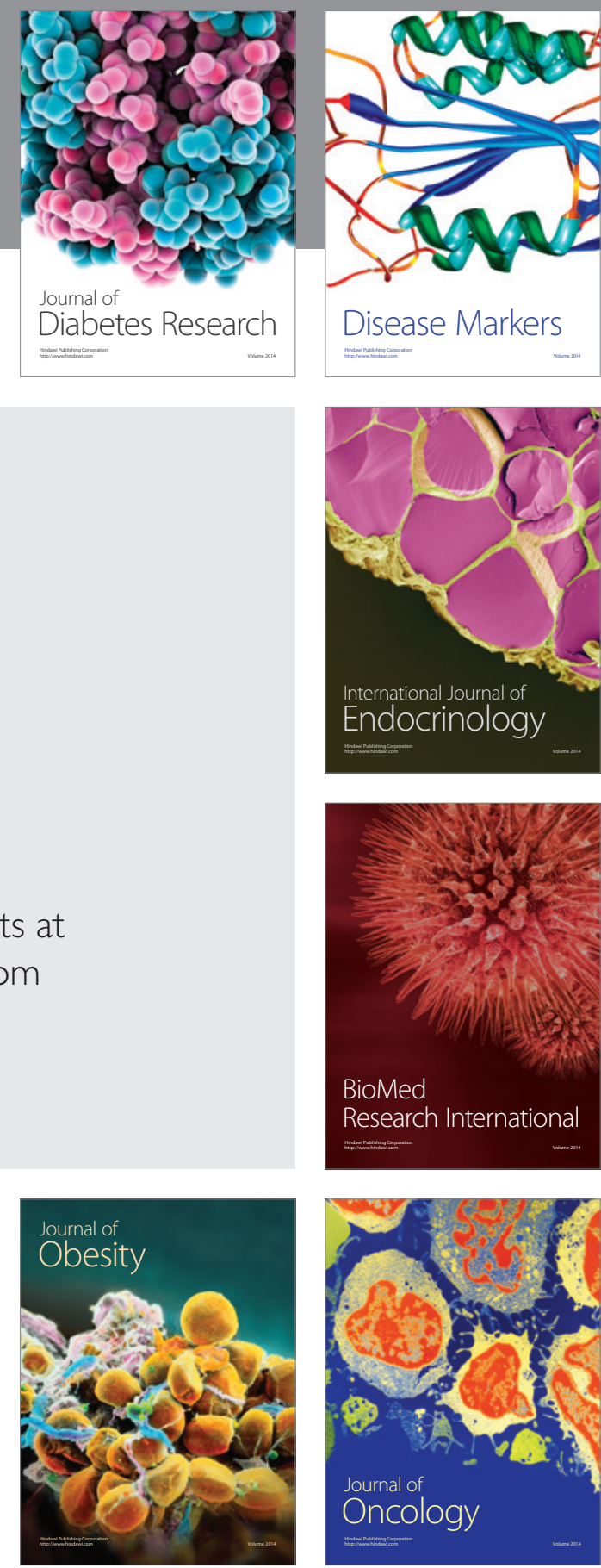

Disease Markers
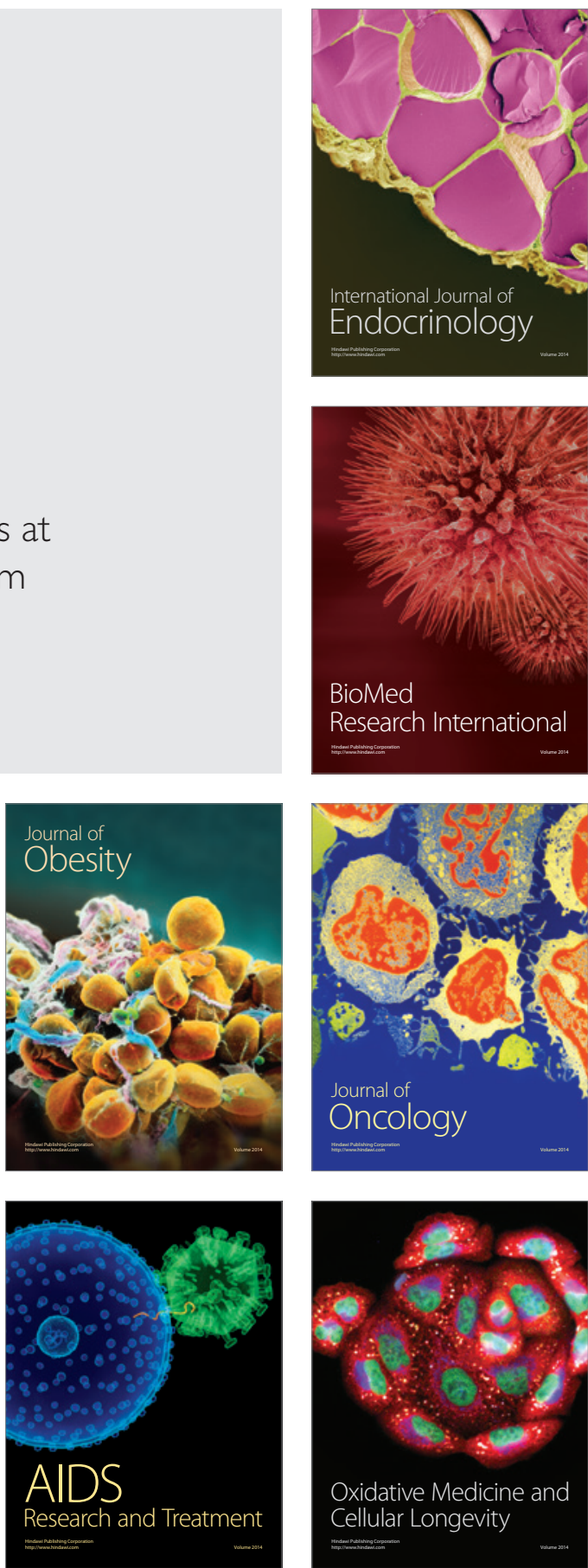